Received: 11 May 2018

Accepted: 26 March 2019

Published online: 16 April 2019
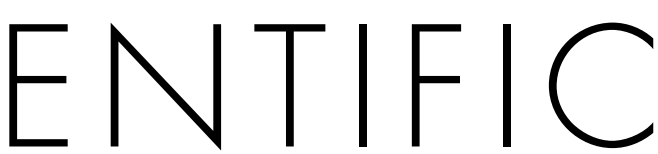

REP

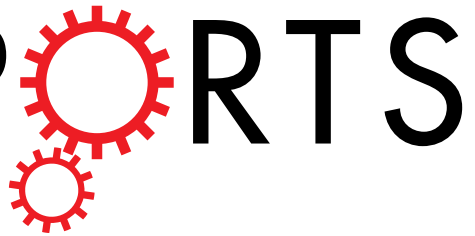

\title{
Structure-based Design of JOC- $x$, a Conjugatable Tumor Tight Junction Opener to Enhance Cancer Therapy
}

Ragan Pitner ${ }^{1,2}$, Jiho Kim ${ }^{1,3}$, Jenn Davis-Bergthold ${ }^{1}$, Cheri Turner ${ }^{1}$, Emilie Vassal-Stermann ${ }^{4}$, Hongjie Wang ${ }^{5}$, Jaclyn Adams ${ }^{5}$, Lauren Carter ${ }^{6}$, Jeffrey A. Ahlgren ${ }^{7}$, Pascal Fender $\mathbb{D}^{4}$, André Lieber ${ }^{5,8}$, Darrick Carter $\mathbb{1}^{1,3,5,8}$ \& Sean A. Gray ${ }^{1}$

Disorganized intercellular junctions are critical for maintaining the integrity of solid epithelial tumors and prevent the infiltration of oncological therapies into the bulk of the malignancy. We have developed small, recombinant proteins which bind a critical junction protein, desmoglein 2, triggering the transient and specific opening of tumor tight junctions allowing for infiltration of the tumor with immune cells, oncolytic viruses, drugs, and other therapeutics. Our new molecule, JOC- $x$, is a promising candidate for a new class of tumor-targeting agents that accumulate both around and within tumors and remodel the tumor microenvironment. Native cysteines were removed from the parental protein, JO-4, followed by addition of a single cysteine to allow for convenient attachment of various payloads that can be targeted directly to the tumor. Our tumor-targeting protein exhibits high avidity, minimal aggregation, and is easily purified at good yields from $E$. coli. For proof of concept, we demonstrate effective conjugation to biotin as a model for flexible co-targeting, addition of metal ion chelators as models for imaging and radiotherapy, and linkage of the TLR3 agonist poly $(\mathrm{l}: \mathrm{C})$ as a model immuneoncologic agent. This second-generation cancer co-therapeutic protein is optimized for activity and primed for CGMP manufacture in preparation for upcoming clinical studies.

There are physical barriers to tumor penetration by cancer drugs. One of the key features of epithelial tumors is the presence of intercellular junctions that link cells to one another and act as barriers to the penetration of molecules with molecular masses greater than four hundred Daltons ${ }^{1-3}$. Several studies have shown that up-regulation of epithelial junction proteins correlate with increased resistance to treatment - including with therapies using monoclonal antibodies and chemotherapeutics ${ }^{4-6}$. One of these junction proteins, desmoglein 2 (DSG2), is a membrane glycoprotein which participates in the formation of tight junctions. Many epithelial cancers are known to highly upregulate the production of DSG2 resulting in formation of a network of cellular "staples" reminiscent of poorly organized junctions that render the tumors resistant to permeation by immune cells and cancer treatments. In one study, levels of DSG2 were found in all of the 60 primary and metastatic breast cancer biopsies analyzed $^{7}$. Moreover, mRNA profiling of ovarian cancer biopsies revealed overexpression of $d s g 2$ message in at least 50 samples analyzed (data not shown). It is thought that the epithelial phenotype of cancer cells and their ability to form physical barriers provides protection from the host immune system and/or elimination by cancer therapeutics ${ }^{8}$.

Junction Opener 1 (JO-1) is a protein that binds to DSG2 and preferentially opens tumor tight junctions ${ }^{7}$ (Fig. 1). JO-1 was derived from the C-terminal knob domain of protein fibers on Adenovirus serotype 3 (Ad3) capsids, which mediates binding of the virus to DSG2 within the tight junctions. This binding initiates a cascade of events within the host cell leading to DSG2 shedding and eventual opening of tight junctions, thus allowing for translocation of the virus between epithelial cells through the basolateral intercellular space ${ }^{9}$. This phenomenon of opening tight junctions provided the basis for developing a co-therapeutic protein to enhance entry of

${ }^{1}$ PAI Life Sciences, Inc., Seattle, WA, USA. ${ }^{2}$ University of Washington, Dept. of Immunology, Seattle, WA, USA. ${ }^{3}$ University of Washington, Program in Pathobiology, Dept. of Global Health, Seattle, WA, USA. ${ }^{4}$ Institut de Biologie Structurale, UMR5075, CNRS/CEA/UGA, Grenoble, France. ${ }^{5}$ University of Washington, Division of Medical Genetics, Seattle, WA, USA. ${ }^{6}$ Institute for Protein Design, Department of Biochemistry, University of Washington, Seattle, WA, USA. ${ }^{7}$ Wyatt Technology Corporation, Santa Barbara, CA, USA. ${ }^{8}$ Compliment Corp., Seattle, WA, USA. Ragan Pitner and Jiho Kim contributed equally. Correspondence and requests for materials should be addressed to S.A.G. (email: sean.gray@pailifesciences.com) 

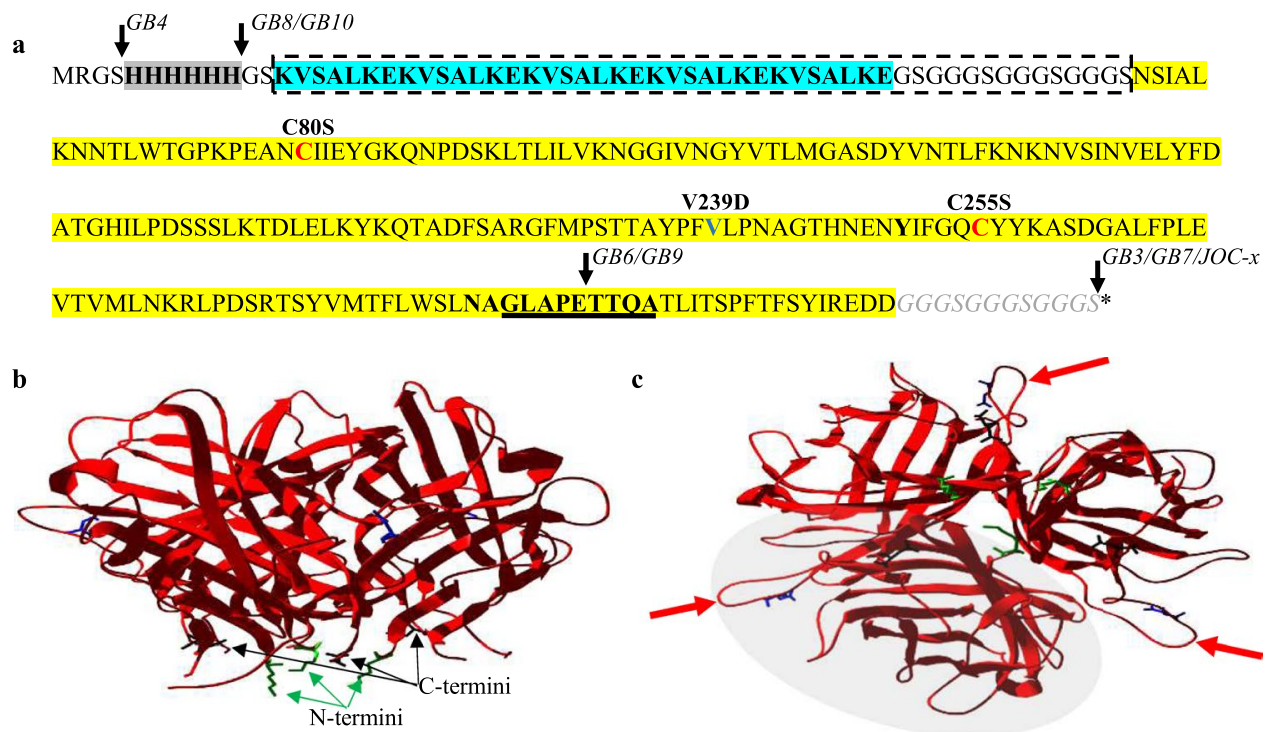

Figure 1. Sequence and structure of JO-1. Panel (a) shows the amino acid sequence of JO- 1 with the $6 \times$ His-tag highlighted in grey, the dimerization domain is shown in bold text and highlighted in light blue, and the Ad3 knob highlighted in yellow. The affinity-enhancing V239D mutation and two internal cysteines at aa80 and aa255 are indicated above their respective residue. The H-I loop is underlined. In dimerization domain-deleted mutants, residues deleted are shown by the dashed box. Cysteinyl residues were inserted at locations shown by black arrows in each GB protein indicated next to the arrow. Panel (b) represents side view of the JO-4 trimer structure indicating the location of the $\mathrm{N}$ - and C-termini. Panel (c) shows a top down view of the trimer with red arrows highlighting the DSG2 binding loop in this view. The grey shaded oval approximates one JO-1 monomer. The structures are adapted from that of PDB Accession \# 1H7Z_A as reported previously ${ }^{11,12}$.

biologics and chemotherapeutic agents such as Doxorubicin, Abraxane, or Irinotecan into tumors. In healthy cells, DSG2 is sequestered to the lateral cell junctions and is minimally available for binding. However, depolarization of tumor cells during epithelial to mesenchymal transition (EMT) leads to localization of DSG2 protein throughout the tumor rather than only within the cell junctions. Additionally, this is coupled with increased DSG2 expression providing an attractive target for our junction openers.

Early attempts at exploiting this property of Ad3 included recombinant expression of the fiber knob domains responsible for DSG2 binding (shown in yellow in Fig. 1a). However, these proteins did not produce the desired tight-junction opening effects possibly due to inability of the knob protein to form active higher-order quaternary structures $^{10,11}$. To overcome this obstacle, a dimerization domain (DD) was added to facilitate multimerization ${ }^{10}$. This domain, a K-coil, is comprised of 5 repeats of 7 amino acids (shown in blue in Fig. 1a). Upon addition of the $\mathrm{DD}$ to the knob protein derived from Ad3, the resulting recombinant protein (JO-1) was able to form multimers, bind DSG2 in vitro, and inhibit Ad3 virus entry into HeLa cells. The JO-1 protein has since been extensively characterized, including the solved crystal structure of the active trimer (Fig. 1b,c). The trimeric form of the protein is the minimal conformation which will bind DSG2, although with low affinity ${ }^{11}$. However, for junction-opening activity, higher-order multimers are required, including the trimer-dimer, which has been described as a barbell structure $^{10,11}$.

Mutagenic libraries of Ad3 fiber knobs identified a panel of mutations conferring higher affinities to DSG2 and enhancing viral inhibition in a functional assay ${ }^{11}$. DSG2 binding by select mutants was measured by surface plasmon resonance (SPR) to determine if the affinity increase was due to faster association rates or more stable dissociation rates ${ }^{11}$. One mutation, substituting the native valine to an aspartic acid at position 239 (V239D), resulted in a nearly 1000 -fold increase in affinity as measured by a shift of the dissociation constant $\left(\mathrm{K}_{\mathrm{D}}\right)$ from $10.8 \mu \mathrm{M}$ for the original JO- 1 to $11.4 \mathrm{nM}$ for the V239D mutant (designated JO-4) ${ }^{11}$. The JO-4 V239D mutation is localized to a region of the knob corresponding to a prominent loop (E-F) known to be critical for stabilizing DSG2 binding (indicated with red arrows in Fig. 1c). An additional eight Ad3 fiber knob mutations in three key regions (C-D loop, F-G loop, and H-I loop) were identified that reduced or ablated DSG2 binding ${ }^{11}$. One mutation in particular, E299V, located in the H-I loop region (underlined in Fig. 1a), was shown to reduce DSG2 binding in the mutant by $80 \%{ }^{11}$.

Preliminary studies in mice have shown these junction openers to be effective at enhancing cancer therapy and very well tolerated even at high doses in non-human primates ${ }^{12}$. This promising in vivo data has resulted in an application as an investigative new drug (IND) for co-therapeutic treatment of certain types of tumors ${ }^{12}$. While co-administration of the JO-4 with effector molecules is an attractive model with substantial scientific support, the ability to produce a conjugatable junction opener with a specific site for covalent attachment to effector molecules would confer further production and deployment advantages. We envision that our designer junction openers would enable nanoparticle delivery to multiple cancers/tumors and may also be useful for chimeric antigen receptor (CAR) T-cell targeting. With respect to cancers, depolarization of cells during EMT as 
well as upregulation in DSG2 expression in tumors relative to normal tissues makes for an attractive target for our molecules.

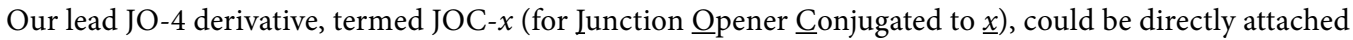
to tumor imaging molecules such as europium or further conjugated with radionuclides for direct ionization of the tumor microenvironment. We investigated direct conjugation of polyriboinosinic:polyribocytidylic acid [poly(I:C)] to JOC- $x$ for targeting and activating TLR3 and MDA5 receptors. By targeting poly(I:C) directly to the tumor-resident dendritic cells (DC), we would mimic natural infection by dsRNA viruses and initiate a robust inflammatory response typified by recruitment and activation of CD8+ T cells. Activation of TLR3 on tumor cells can also lead to tumor killing by initiating apoptosis, as has been demonstrated with the HPV vaccines BiVax and $\operatorname{TriVax}^{13,14}$. Moreover, numerous cancers are characterized by upregulation of TLR3, a receptor for poly(I:C), and in most of these cancers this TLR3 positivity is predictive of a favorable outcome if poly(I:C) is used as an immune therapeutic ${ }^{13-18}$. Given the ability of JOC- $x$ to specifically localize to tumors overexpressing DSG2 and the known anti-tumor effects of poly(I:C), we envision that the JOC-poly(I:C) conjugate would exhibit enhanced targeting of tumors, increased potency, and overall less toxicity due to reduced binding to healthy tissues expressing TLR3 receptors and DSG2 that is properly sequestered in tight junctions.

We report here development, characterization, and in vitro validation of a new junction opening molecule, JOC- $x$. Preliminary characterization of JOC- $x$ reveals many attractive features for production, manufacture, co-administration, and conjugated targeting making it a promising candidate to move towards human trials.

\section{Results}

Generation of a protein without internal cysteines and with a free cysteine for conjugation. JO-1 has two native cysteinyl residues (amino acids 80 and 255) which, based on the crystal structure, are spatially distant and unlikely to form intramolecular disulfide bridges. Ten JO-1 derivatives (termed GB1-10) were generated, with six containing the dimerization domain and four in which the dimerization domain was eliminated to interrogate the effects on multimerization and DSG2 binding activity. Supplementary Fig. 1 shows a pictorial representation of each GB protein compared to JO-1, with key changes highlighted. GB1 is a codon optimized protein identical to JO-1 but optimized to maximize expression in E. coli. GB2 was constructed by mutating the native internal cysteines of GB1 to serinyl residues to limit oxidation and covalent aggregation. Next, we reintroduced a single cysteine to act as a designer sulfhydryl-based site for targeted conjugation at various locations within the proteins as follows: in GB3 the cysteine was added at the $\mathrm{C}$-terminus following a flexible glycine serine linker $\left[\left(\mathrm{G}_{3} \mathrm{~S}\right)_{3}\right]$; in GB4 and GB10, the single cysteinyl residue was added at the N-terminus either before (GB4) or immediately following (GB10) the His tag; and for GB6, the cysteine was introduced proximal to the glutamic acid residue at position aa 299 within the H-I loop (PECTT, underlined in Fig. 1a), a key region known to stabilize DSG2 binding.

The free cysteine functions as a covalent mediator of multimerization. The DD contains 5 repeats of seven amino acids, with two lysine residues per repeat, which we predicted could potentially result in ribosomal stalling during translation and lower protein yields. Addition of the DD, with its predicted isoelectric point $(p \mathrm{I})$ of $\sim 10.91$, to the Ad3 knob results in an increase in predicted $p \mathrm{I}$ from $\sim 7.32$ (Ad3 knob) to $\sim 8.65$ (JO-1). We therefore hypothesized that removal of the DD would likely (1) lower aggregation beyond the levels observed with the cysteine-deleted GBs, (2) enhance protein expression levels, and (3) return the protein to a neutral $p \mathrm{I}$ leading to lower residual endotoxins following purification. To test this hypothesis, GB5, GB7, GB8 and GB9 were all designed without the DD and a single cysteine inserted as follows: GB5 contained no cysteine; GB7 was constructed similar to GB3 with the C-terminal $\left(\mathrm{G}_{4} \mathrm{~S}\right)_{3}$ followed by a terminal cysteine residue; GB8 contained a cysteine immediately following the His tag; and GB9 was similar to GB6 with a cysteine in the H-I loop (Supplementary Fig. 1).

Elimination of the dimerization domain confers multiple production advantages. Nine of the ten GB proteins produced in E. coli. The exception, GB6, failed to express stable protein and was eliminated from further analysis. Expression levels of GBs containing the DD ranged from $0.5-5 \mathrm{mg} / \mathrm{L}$, similar to JO- 1 , whereas clones without the DD were 4-10-fold higher and ranged from 5-20 mg/L. Endotoxin levels of clones containing the DD ranged from 80,000 to $\sim 1,500,000 \mathrm{EU} / \mathrm{mg}$ with an average of $691,300 \mathrm{EU} / \mathrm{mg}$ as determined using the Limulus Amebocyte Lysate (PTS-LAL) assay (Charles River Laboratories, Inc., Charleston, SC). In addition, endotoxin was very difficult to remove without significant protein losses. In contrast, endotoxin levels of DD-deleted clones were 100- to 1000-fold lower ranging from 3000-20000 EU/mg. Production chromatography of GB7 (with no DD and a single C-terminal cysteine) resulted in an average endotoxin level of only $2500 \mathrm{EU} / \mathrm{mg}$ which was easily reduced to $<10 \mathrm{EU} / \mathrm{mg}$ following a single pass through an endotoxin removal column.

Functional responses are preserved. Each of the GB proteins migrated according to their expected molecular weight on boiled and reduced SDS-PAGE (Supplementary Fig. 2a). We assessed multimerization using samples that were not reduced and not boiled prior to SDS-PAGE (Supplementary Fig. 2b), which-while not true native analysis-allows this family of proteins to retain multimeric forms even in the presence of SDS detergent. This was done because previous data had indicated that denatured, improperly folded, or otherwise inactivated JO-1 does not form higher-order multimers on non-reduced SDS-PAGE, does not bind DSG2, and does not inhibit viral entry ${ }^{11}$. As shown in Supplementary Fig. $2 \mathrm{~b}$, several multimeric states were observed including monomers (M), trimers (T), trimer-dimers (T-D), and multimers/aggregates (Agg.) at $>150 \mathrm{kDa}$. In the non-reducing gel, all nine GB proteins formed higher order multimers, although a significant percentage of GB9 maintained a monomeric state. GB5 appeared to form trimers but did not produce higher MW isoforms such as trimer-dimers. 


\begin{tabular}{|l|l|l|}
\hline Protein & DSG2 binding & VIA IC $_{\mathbf{5 0}}(\boldsymbol{\mu} \mathbf{g} / \mathbf{m L})$ \\
\hline JO-4 & Yes & 0.067 \\
\hline GB1 & Yes & 0.025 \\
\hline GB2 & Yes & 0.030 \\
\hline GB3 & Yes & 0.029 \\
\hline GB4 & Yes & 0.037 \\
\hline GB5 & Minimal & 0.241 \\
\hline GB6 & n/a & $\mathrm{n} / \mathrm{a}$ \\
\hline GB7/JOC- $x$ & Yes & 0.032 \\
\hline GB8 & Yes & 0.012 \\
\hline GB9 & No & $>10$ \\
\hline GB10 & Yes & 0.026 \\
\hline
\end{tabular}

Table 1. Phenotype of $\mathrm{JO} 4$ derivatives.

\begin{tabular}{|l|l|l|l|}
\hline Protein & $\mathbf{k}_{\mathbf{a}}(\mathbf{M} / \mathbf{s})$ & $\mathbf{K}_{\mathbf{d}}(\mathbf{1} / \mathbf{s})$ & $\mathbf{K}_{\mathbf{D}}(\mathbf{n M})$ \\
\hline a. Rates following immobilization of hDSG2 protein \\
\hline JO4 & $2.32 \times 10^{5}$ & $2.64 \times 10^{-3}$ & 11.4 \\
\hline $\mathrm{GB}_{\mathrm{V} 239 \mathrm{D}}$ & $\mathbf{4 . 4} \times \mathbf{1 0}^{\mathbf{6}}$ & $5.0 \times 10^{-4}$ & $\mathbf{0 . 1 1}$ \\
\hline $\mathrm{GB}_{\mathrm{V} 239 \mathrm{D}} / \mathrm{JOC}-x$ & $1.4 \times 10^{6}$ & $8.3 \times 10^{-4}$ & 0.58 \\
\hline b. Rates following immobilization of junction opener proteins \\
\hline JO4 & $7.9 \times 10^{5}$ & $\mathbf{4 . 6} \times \mathbf{1 0}^{-\mathbf{4}}$ & 0.58 \\
\hline $\mathrm{GB}_{\mathrm{V} 239 \mathrm{D}}$ & Not tested & 1.19 \\
\hline $\mathrm{GB}_{\mathrm{V} 239 \mathrm{D}} / \mathrm{JOC}-x$ & $6.3 \times 10^{5}$ & $7.5 \times 10^{-4}$ \\
\hline
\end{tabular}

Table 2. Biacore analysis of binding to hDSG-2.

To confirm the presence of active conformations, the panel was tested for binding to human DSG2 by Western analysis probing with labeled hDSG2 (Supplementary Fig. 2c). Except for GB5 and GB9, prominent DSG2 binding was visible in the region of the blot corresponding to trimers, timer-dimers, and aggregates, but not at the size corresponding to monomers. This result was not unexpected for GB5 given the lack of higher MW multimers beyond the trimer. GB9, despite forming multiple higher order multimers, failed to bind DSG2 confirming earlier reports on the importance of the H-I loop as one of three key regions required for DSG2 binding by the Ad3 $\mathrm{knob}^{11,19}$.

We next assessed the ability of the GBs to bind to DSG2 in vitro using HeLa cells in a competition-based viral inhibition assay (VIA) (Supplementary Fig. 2d) ${ }^{11}$. This assay determines the minimum protein concentration required to inhibit 50\% entry of a fluorescent $\mathrm{Ad} 3$ reporter virus and is a robust predictor of in vivo activity. These viral inhibition curves can be fit to a 5-parameter curve from which the concentration at which $50 \%$ of viral entry is inhibited $\left(\mathrm{IC}_{50}\right)$ can be calculated and reported in $\mu \mathrm{g} / \mathrm{mL}$. Results of DSG2 binding and $\mathrm{IC}_{50}$ values for the ten GBs are listed in Table 1. Seven of the nine GBs had binding curves similar to JO-1, with GB5 showing significantly lower and GB9 showing almost no Ad3 virus inhibition.

Down-selection of next generation conjugatable junction openers. Based on production levels, DSG2 binding, and viral inhibition results, we down-selected GB3 and GB7 for further characterization of multimerization. To improve binding kinetics of GB3 and GB7, we introduced the JO-4 mutation by substituting a valine at position 239 for an aspartic acid resulting in the high affinity versions GB3 $3_{\mathrm{V} 239 \mathrm{D}}$ and $\mathrm{GB} 7_{\mathrm{V} 239 \mathrm{D}}$. We prioritized GB7 over GB8 since having a cysteine at the C-terminus would be advantageous for conjugation of the protein to effector molecules (drugs, antibodies, poly(I:C), or isotopes) as well as delivery systems such as nanoparticles. With respect to GB8, we also hypothesized that conjugation to a cysteine proximal to the $6 \times$ his tag could pose steric hindrances which could affect downstream purification efforts.

Domains of DSG2 are cleaved and shed by the action of matrix metalloproteinases in a process that is enhanced by treatment with our junction opener constructs. This shed DSG2 can be quantified using a simple ELISA $^{20}$. Over the course of 48 hours, JO-4, GB3 ${ }_{\mathrm{V} 239 \mathrm{D}}$, or GB7 ${ }_{\mathrm{V} 239 \mathrm{D}}$ all resulted in increased DSG2 shedding compared to untreated cells (data not shown). For a more accurate measurement of DSG2 binding, we compared the avidities and association/dissociation rates of JO-x derivatives to DSG2. Surface plasmon resonance (SPR) analysis was performed by immobilizing recombinant human DSG2 (rhDSG2) onto a Biacore sensor chip and flowing JO-4, GB3 ${ }_{\mathrm{V} 239 \mathrm{D}}$, or GB7 $\mathrm{V} 239 \mathrm{D}_{\mathrm{D}}$ across the surface. A roughly 20 -fold more avid binding of DSG2 was determined for $\mathrm{GB}_{\mathrm{V} 239 \mathrm{D}}$ compared to JO-4 $\left(0.58 \mathrm{nM}\right.$ and $11.4 \mathrm{nM}$, respectively). This apparent higher affinity of GB7 $7_{\mathrm{V} 239 \mathrm{D}}$ appeared to be due to both a faster association rate and slower dissociation rate compared to JO-4 (Table 2a). Of the three proteins, $\mathrm{GB}_{\mathrm{V} 239 \mathrm{D}}$ had the highest affinity of $0.11 \mathrm{nM}$ primarily due to a nearly one $\log$ faster association rate of $4.4 \times 10^{6} \mathrm{M} / \mathrm{s}$ compared to JO-4 and nearly 3-fold faster on-rate compared to $\mathrm{GB}_{\mathrm{V} 239 \mathrm{D}}$. In this format, both JO-4 and the GB3 ${ }_{\mathrm{V} 239 \mathrm{D}}$ proteins appeared to bind to the sensor surface somewhat non-specifically and were more difficult to regenerate from the sensor surface compared to GB7 ${ }_{\mathrm{V} 239 \mathrm{D}}$. We hypothesized that presence of 

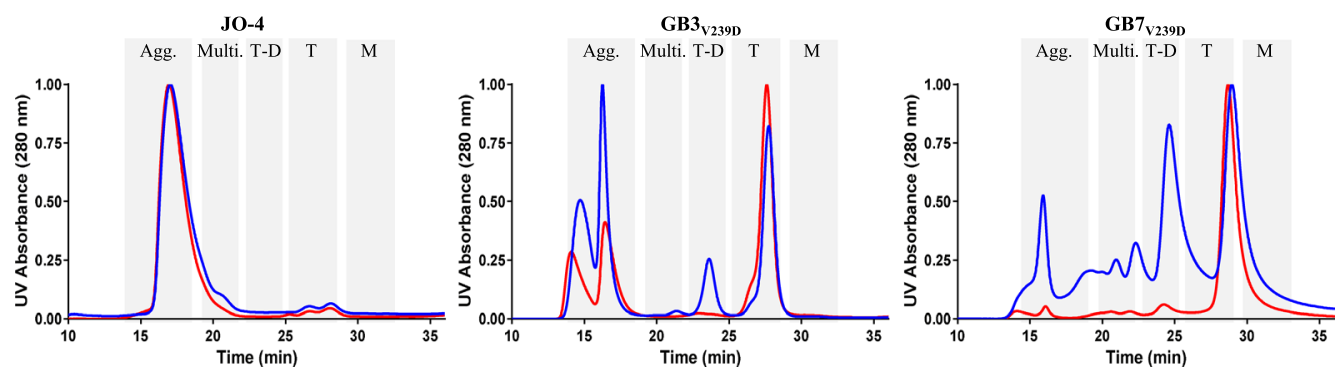

Figure 2. Distribution of protein multimerization states determined by SEC analysis. The SEC plots for JO-4 (left), GB3 $3_{\mathrm{V} 239 \mathrm{D}}$ (middle), and GB7 ${ }_{\mathrm{V} 239 \mathrm{D}}$ (right). The blue line represents the A280 absorbance for the oxidized protein while the red line represents the reduced protein. The $y$-axis represents the approximate fraction of each peak while the $x$-axis shows the elution time in minutes. The grey shaded boxes indicate the approximate retention time for (Agg) aggregates, (Multi) multimers, (T-D) trimer-dimers, (T) trimers, and (M) monomers based on MALS analysis.

\begin{tabular}{|c|c|c|c|c|c|c|c|c|}
\hline \multirow{3}{*}{$\begin{array}{l}\text { Approx. } \\
\text { Elution } \\
\text { Time (min) }\end{array}$} & \multirow{3}{*}{$\begin{array}{l}\text { Approx. } \\
\text { MW (kDa) }\end{array}$} & \multirow[b]{3}{*}{ Likely species } & \multicolumn{6}{|c|}{ \% total of each species } \\
\hline & & & \multicolumn{2}{|c|}{ JO4 } & \multicolumn{2}{|l|}{$\mathrm{GB3}_{\mathrm{V} 239 \mathrm{D}}$} & \multicolumn{2}{|c|}{$\mathrm{GB}_{\mathrm{V} 239 \mathrm{D}} / \mathrm{JOC}-x$} \\
\hline & & & Oxidized & Reduced & Oxidized & Reduced & Oxidized & Reduced \\
\hline $13.8-18.2$ & $3000-578$ & Aggregate (Agg.) & 90 & 94 & 55 & 43 & 12 & 4 \\
\hline $19.2-21.5$ & 334-289 & Multimer (Multi.) & 4 & $<0.5$ & 2 & $<0.5$ & 23 & 4 \\
\hline $22.2-24.6$ & $167-144$ & Trimer-Dimer (T-D) & $<0.5$ & 1 & 10 & 3 & 31 & 2 \\
\hline $25.0-28.3$ & $83-72$ & Trimer $(\mathrm{T})$ & 2 & 2 & 33 & 53 & 34 & 89 \\
\hline $29.2-32.4$ & $24-28$ & Monomer (M) & 4 & 3 & $<0.5$ & $<0.5$ & $<0.5$ & $<0.5$ \\
\hline
\end{tabular}

Table 3. Distribution of protein multimerization states determined by MALS analysis.

positively charged residues within the $\mathrm{DD}$ of $\mathrm{JO}-4$ and $\mathrm{GB} 3_{\mathrm{V} 239 \mathrm{D}}$ may have resulted in binding to the negatively charged sensor surface and that the greater abundance of multimers and aggregates, more prominent in JO-4 and $\mathrm{GB}_{\mathrm{V} 239 \mathrm{D}}$, may have retarded diffusion of these molecules compared to GB7 $\mathrm{V} 239 \mathrm{D}$. To minimize non-specific binding that may be due to the $\mathrm{DD}$, we repeated the assay by immobilizing one protein containing the $\mathrm{DD}$ (JO-4) and one without the DD (GB7 $\left.{ }_{\mathrm{V} 239 \mathrm{D}}\right)$ while flowing DSG2 across the surface $\left(\mathrm{GB} 3_{\mathrm{V} 239 \mathrm{D}}\right.$ was not tested in this format). In this format, the apparent avidity of JO-4 to DSG2 improved from $11.4 \mathrm{nM}$ to $0.58 \mathrm{nM}$, while the affinity of GB7 $_{\mathrm{V} 239 \mathrm{D}}$ for DSG2 was nearly identical albeit slightly reduced (Table 2b).

Junction openers without the dimerization domain exhibit minimal aggregation. To investigate the quaternary structure of the proteins, we performed size-exclusion chromatography and multi-angle light scattering analysis (SEC-MALS) on JO-4, GB3 ${ }_{\mathrm{V} 239 \mathrm{D}}$, or $\mathrm{GB}_{\mathrm{V} 239 \mathrm{D}}$. Each protein was tested in the native (presumably oxidized) form or following treatment with low levels of dithiothreitol (DTT) to reduce disulfide bonds. The UV traces of these analyses are shown in Fig. 2 whereby the shaded and labeled boxes indicate where the aggregates (Agg.), multimers (Multi.), trimer-dimers (T-D), trimers (T), or monomers (M) eluted based on size determination by the MALS detector.

The MALS analyses - including percentage of each species, molecular weights, and elution times - are summarized in Table 3. In its oxidized state, JO-4 presented a dominant peak in the region corresponding to aggregates with approximately $94 \%$ existing as aggregates or multimers and a minority existing as monomers or trimers. Less than $1 \%$ existed as trimer-dimers. Reduction of JO-4 with DTT did not significantly alter the aggregation phenotype. Removal of internal cysteines in $\mathrm{GB}_{\mathrm{V} 239 \mathrm{D}}$ significantly reduced the aggregation phenotype with $57 \%$ of the protein existing as aggregates or multimers, $10 \%$ existing as trimer-dimers, and 33\% existing as trimers. Reduction of $\mathrm{GB}_{\mathrm{V} 239 \mathrm{D}}$ resulted in a slight reduction in aggregates, a 3-fold reduction in trimer-dimers, and significant increase of trimers from $33 \%$ to $53 \%$. In the oxidized state, GB7 $7_{\mathrm{V} 239 \mathrm{D}}$ existed as trimers (34\%) and $31 \%$ as trimer-dimers, with $35 \%$ existing as multimers, or aggregates. Upon reduction, the percentage of trimers significantly increased (89\%) with a concomitant reduction in all three higher order species to a total of $10 \%$. This is consistent with the engineered cysteinyl residue performing as expected allowing for targeted multimerization which can be reversed by reduction. For all 3 proteins, only a small amount was found in their monomeric form even after reduction. This agrees with un-boiled, un-reduced SDS-PAGE analysis of GB7 (Supplementary Fig. 2b) in which almost no visible monomers are observed even in the presence of reducing and denaturing agent.

Further investigation of the quaternary structure was performed using uranyl acetate negative staining of protein smears and imaging by transmission electron microscopy (TEM ${ }^{21}$. As shown in Fig. 3a, JO- 4 exhibited relatively uniform higher order structures approaching $50 \mathrm{~nm}$ in diameter (white arrows) that resemble the penton-dodecahedra (Pt-Dd) described previously ${ }^{9,22}$. Penton-dodecahedra are believed to be made up of 12 pentons of pentameric base and 12 trimers of the fiber knob with a predicted mass of at least $1253 \mathrm{kDa}$. This is similar to the size measured by MALS for the JO-4 aggregates, estimated to average $1120 \mathrm{kDa}$ in the oxidized state. By 


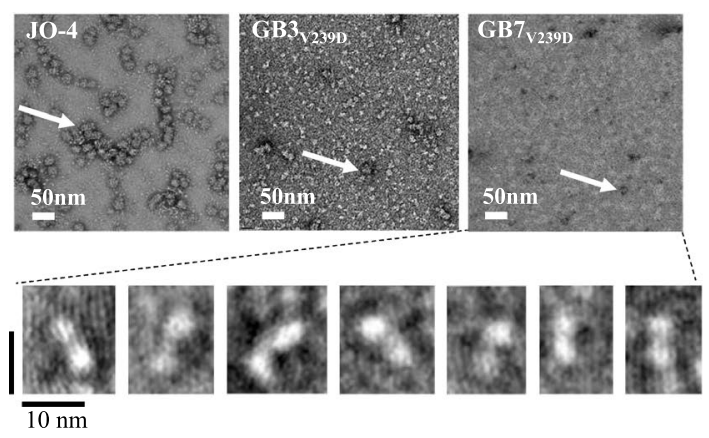

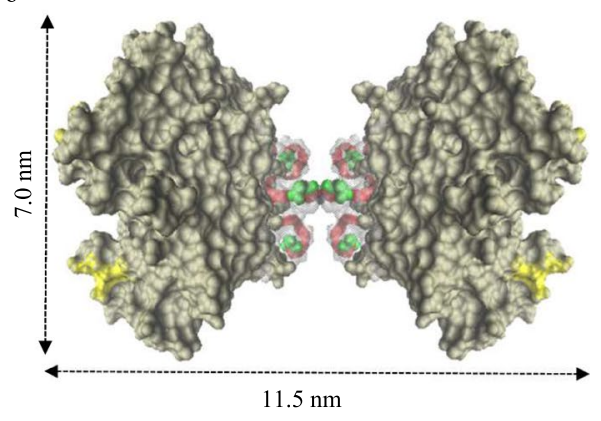

Figure 3. TEM images of protein aggregation and trimer-dimer formation. Panel (a) uranyl acetate negative staining of protein for JO-4 (left), GB3 $\mathrm{V} 239 \mathrm{D}$ (middle), and GB7 $\mathrm{V} 239 \mathrm{D}_{\mathrm{D}}$ (right). The white arrows indicate aggregates which appear nearly $50 \mathrm{nM}$ in diameter according to the $50 \mathrm{nM}$ size bar in white at the lower left of the figure. Panel (b) the space-filling model of the trimer-dimer of JO-4 was created in VMD 23 using the known x-ray crystallographic coordinates of JO-4. The model depicts a barbell shape with an approximate length of $11.5 \mathrm{~nm}$ and a width of $7 \mathrm{~nm}$. Panel (c) fine resolution enhancement images of white specks seen in the GB7 ${ }_{\mathrm{V} 239 \mathrm{D}}$ image in panel a. The images reveal structures resembling the barbell structure in both size and shape of the modeled trimer-dimer.

TEM, GB3 ${ }_{\mathrm{V} 239 \mathrm{D}}$ exhibits fewer high MW aggregates compared to JO-4. This observation is even more pronounced with $\mathrm{GB}_{\mathrm{V} 239 \mathrm{D}}$ as only a few aggregates are observable in the image field. The white specks in the background and prevalent throughout the images were presumed to be trimers and/or trimer-dimers, the latter of which have been described as resembling barbell-like structures. Using the Visual Molecular Dynamics program (VMD, U. of Illinois Urbana-Champaign $)^{23}$ and the known X-ray crystallographic coordinates for JO-4, we modeled the trimer-dimer structure as shown in Fig. 3b. The resulting trimer-dimer structure indeed appears barbell-shaped with approximate dimensions of $7 \mathrm{~nm}$ wide by $11.5 \mathrm{~nm}$ long. Fine resolution enhancement of seven of these white specks from the GB7 ${ }_{\mathrm{V} 239 \mathrm{D}}$ image are shown in Fig. 3c. These specs clearly resembled the barbell-shape of the modeled trimer-dimer (Fig. 3b) in both size and shape.

Junction openers with alternate multimerization strategies maintain full viral inhibition. As mentioned above, SEC-MALS analysis suggested that reduction had almost no impact on JO-4 multimerization, minimal impact on $\mathrm{GB}_{\mathrm{V} 239 \mathrm{D}}$ multimerization, and a pronounced impact on $\mathrm{GB} 7_{\mathrm{V} 239 \mathrm{D}}$ multimerization. It has been shown that the junction openers will only open tight junctions or inhibit viral entry as a trimer-dimer, multimer, or aggregate ${ }^{10,11}$. Moreover, trimers, but not monomers, will bind DSG2 but will not open tight junctions $^{10,11}$. We therefore sought to test the ability of JO-4, GB3 ${ }_{\mathrm{V} 239 \mathrm{D}}$, and GB7 ${ }_{\mathrm{V} 239 \mathrm{D}}$ to inhibit Ad3 viral entry in the reduced or oxidized state. Based on SEC-MALS analysis, we predicted that JO-4 would show equivalent levels of viral inhibition given that it maintains higher order structures following reduction. However, we expected GB7 $_{\text {V239D }}$ to exhibit the greatest loss in viral inhibition given that MALS analysis showed that $\sim 89 \%$ of the protein was found as trimers following reduction. Results of the viral inhibition assay on reduced and non-reduced proteins are shown in Supplementary Fig. 3a while the IC50 values are shown in Supplementary Fig. 3b. As expected for JO-4, oxidized and reduced inhibition curves were nearly indistinguishable from one another and resulted in nearly identical $\mathrm{IC}_{50}$ values. For $\mathrm{GB}_{\mathrm{V} 239 \mathrm{D}}$, reduction resulted in an approximately two-fold decrease in viral inhibition likely due to loss of some aggregates and the three-fold reduction in trimer-dimers estimated by MALS analysis. As expected with $\mathrm{GB}_{\mathrm{V} 239 \mathrm{D}}$, reduction with DTT resulted in a 5-fold decrease in viral inhibition. This was likely due to the 4 -fold loss of aggregates and multimers (from $35 \%$ to $8 \%$ ) and the 15 -fold loss of trimer-dimers (from $31 \%$ to $2 \%$ ) upon reduction as measured by MALS analysis (Table 3 ).

Conjugation of JOC- $x$ with novel payloads. Based on the biochemical and biophysical characterization of JO-4, GB3/GB3 ${ }_{\mathrm{V} 239 \mathrm{D}}$, and GB7/GB7 $\mathrm{V} 239 \mathrm{D}$, we selected $\mathrm{GB} 7_{\mathrm{V} 239 \mathrm{D}}$ as our lead molecule and renamed it JOC- $x$ for Iunction $\underline{\text { Opener }} \underline{\mathrm{C}}$ njugated to $\underline{x}$ to better describe its functional attributes. We then sought to demonstrate the utility of JOC- $x$ as a reagent for pre-targeted radioimmunotherapy (PRIT), direct tumor imaging, or immune therapy ${ }^{24-26}$. The basis of PRIT involves injection of a biotinylated tumor-targeting molecule, allowing the molecule to bind its target, and then forming a complex at the site of the tumor following a second injection of streptavidin conjugated to either an imaging agent or radionuclide. We biotinylated JOC- $x$ at the terminal cysteinyl residue using the EZ Link Maleimide-PEG2 Biotin or PEG11 Biotin kits (Thermo Scientific, Rockford, IL.). The purpose for the PEG2 or PEG11 spacers was to assess whether biotinylation would impair ability of the monomeric protein to form bioactive multimers, and if so, would increasing the linker length reduce steric hindrance and increase multimerization and activity. Following biotinylation, the JOC- $x$ and JOC-PEG2/PEG11 conjugates were tested for viral inhibition as described (Fig. 4a). JOC-PEG2-biotin and JOC-PEG11-biotin both inhibited virus with only slightly impaired $\mathrm{IC}_{50}$ values of 0.125 and $0.203 \mu \mathrm{g} / \mathrm{mL}$, respectively, compared to $0.032 \mu \mathrm{g} / \mathrm{mL}$ for the unconjugated JOC- $x$. As shown in the Coomassie stained panel in Fig. 4b, conjugation of either PEG2or PEG11-biotin did not impair the ability of JOC- $x$ to form multimers as evidenced by the formation of the bands in the Trimer (T) and Trimer-Dimer (T-D) range of the unreduced samples. To further functionalize the JOC-PEG-biotin conjugates, we added a streptavidin-europium (SA-Eu) moiety to the conjugate and analyzed 
a

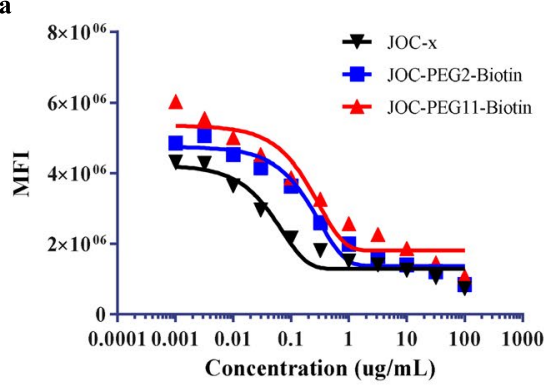

b

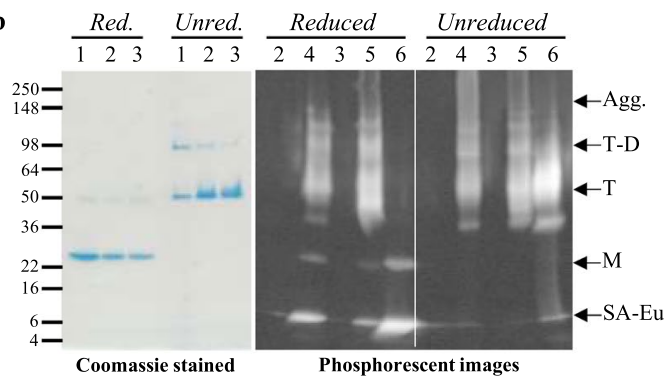

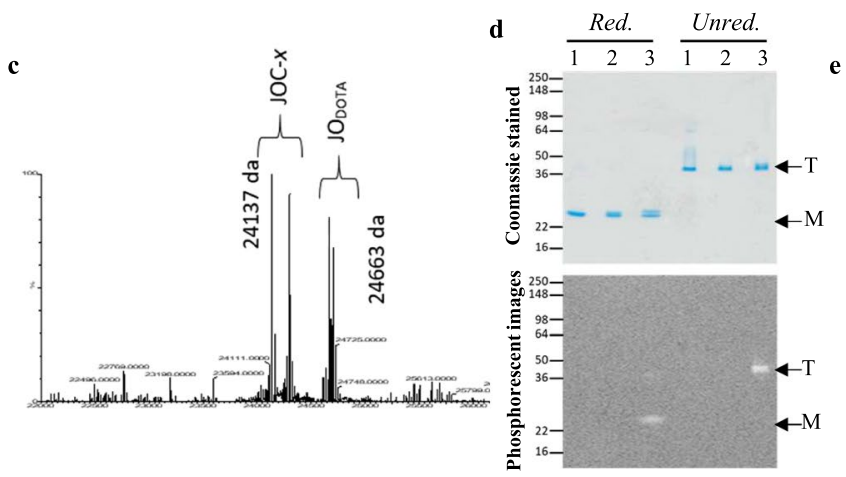

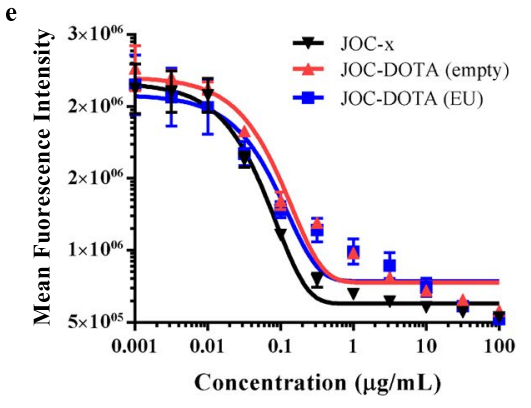

Figure 4. Conjugation of JOC- $x$ with PEG-biotin or DOTA-EU. Panel (a) Shows the viral inhibition curves for JOC- $x$ before and after conjugating to PEG2- or PEG11-biotin. Panel (b) JOC- $x$ and JOC-Biotin conjugates were mixed with streptavidin-europium (SA-Eu) and resolved by SDS-PAGE with or without boiling or reduction by DTT. One gel was stained with Coomassie to visualize formation of multimers and one gel transferred to a PVDF membrane and imaged using a Spectramax I3x Imager to visualize Eu phosphorescence at an emission spectrum of $616 \mathrm{~nm}$. Multimeric forms are indicated to the right of the gel/ blot whereby $\mathrm{SA}-\mathrm{Eu}=$ free streptavidin-europium, $\mathrm{M}=$ Monomer, $\mathrm{T}=$ Trimers, $\mathrm{T}-\mathrm{D}=$ Trimer-Dimers, and Agg. $=$ Aggregates/multimers. Numbering of lanes is as follows: $1=$ JOC- $x, 2=$ JOC-PEG2-biotin, $3=$ JOCPEG11-biotin, 4 = JOC-PEG2-biotin-SA-Eu, $5=$ JOC-PEG11-biotin-SA-Eu, $6=$ SA-Eu alone. Panel (c) A mass spectrometry spectrum for JOC-DOTA is shown indicating the position of unconjugated JOC- $x(24137 \mathrm{Da})$ and JOC-DOTA (24663 Da). Panel (d) Unconjugated JOC- $x$ (1), JOC-DOTA (empty) (2), and JOC-DOTA (Eu) (3) were resolved by SDS-PAGE, with or without boiling or reduction by DTT. One gel was stained with Coomassie (top gel) to visualize formation of multimers and one gel transferred to a PVDF membrane and imaged using a Spectramax I3x Imager (lower blot) as described. Positions of Trimers "T" and monomers "M" are noted to the right of the gel/blot. Panel (e) Viral inhibition curves of JOC- $x$, JOC-DOTA (Empty), and JOC-DOTA (Eu) are shown indicating that DOTA-Eu conjugation only minimally reduces DSG2-mediated viral entry.

their ability to form trimers and trimer-dimers even with the bulky SA-Eu groups attached (Fig. 4b). Europium is a rare earth metal and was chosen for two reasons: (1) it is often used in in vivo imaging due to its phosphorescent properties; and (2) it is a non-radioactive surrogate for radionuclides that may later be used for direct imaging or irradiation of tumors. The phosphorescent panel in Fig. $4 \mathrm{~b}$ shows that even with successful loading of SA-Eu to both PEG2 and PEG11 biotin conjugated JOC- $x$ they were able for form trimers, trimer-dimers, and aggregates (indicated by $T, T-D$, and $A g g$ on Fig. $4 \mathrm{~b}$ ). An inhibition assay with the JOC-PEG-SA-Eu conjugates resulted in atypical curves of viral inhibition that could not be fit with our typical 5-parameter fit. This is likely due to multiple binding interactions taking place simultaneously between SA multimers, SA-JOC-PEG-biotin, and JOC-PEG-SA-Eu to the DSG2 protein on cells. However, we do not consider this problematic given that the full conjugate-payload, JOC-PEG-SA-Eu, would not be directly injected into an animal, but rather would be used stepwise (as described above for PRIT).

A different methodology to generate a directly labeled, less bulky, and more flexible tumor imaging and radiotherapy agent involved conjugation to 1,4,7,10-tetraazacyclododecane-1,4,7,10-tetraacetic acid (DOTA) to the JOC- $x$ resulting in JOC-DOTA. DOTA/Eu labeled proteins have been used extensively for in vivo imaging ${ }^{27-29}$ and also have the advantage of being able to sequester radionuclides, such as yttrium-90, which may be used to irradiate their targets ${ }^{30-33}$. Because of the small size of DOTA $(526 \mathrm{Da})$, we used mass spectrometry to confirm that the DOTA molecule was attached to JOC- $x$. Figure 4c shows a mixture of unconjugated JOC- $x$ and JOC-DOTA with their respective spectra at $24137 \mathrm{Da}$ and $24663 \mathrm{Da}$, respectively.

The JOC-DOTA was then complexed with europium ions to form JOC-DOTA (Eu) and successful labeling was demonstrated by reduced and non-reduced SDS-PAGE (Fig. 4d). Coomassie staining (top gel) showed that JOC- $x$, JOC-DOTA (empty), and JOC-DOTA (Eu) all were able to form trimers in the unreduced analysis. A second, identical gel was transferred to a PVDF membrane and imaged using a Spectramax I3x Imager (lower blot) to capture europium phosphorescence. As shown in Fig. 4d, only the JOC-DOTA (Eu) produced phosphorescence at the monomer $(\mathrm{M})$ position in the reduced gel and the trimer $(\mathrm{T})$ position in the unreduced gel. 

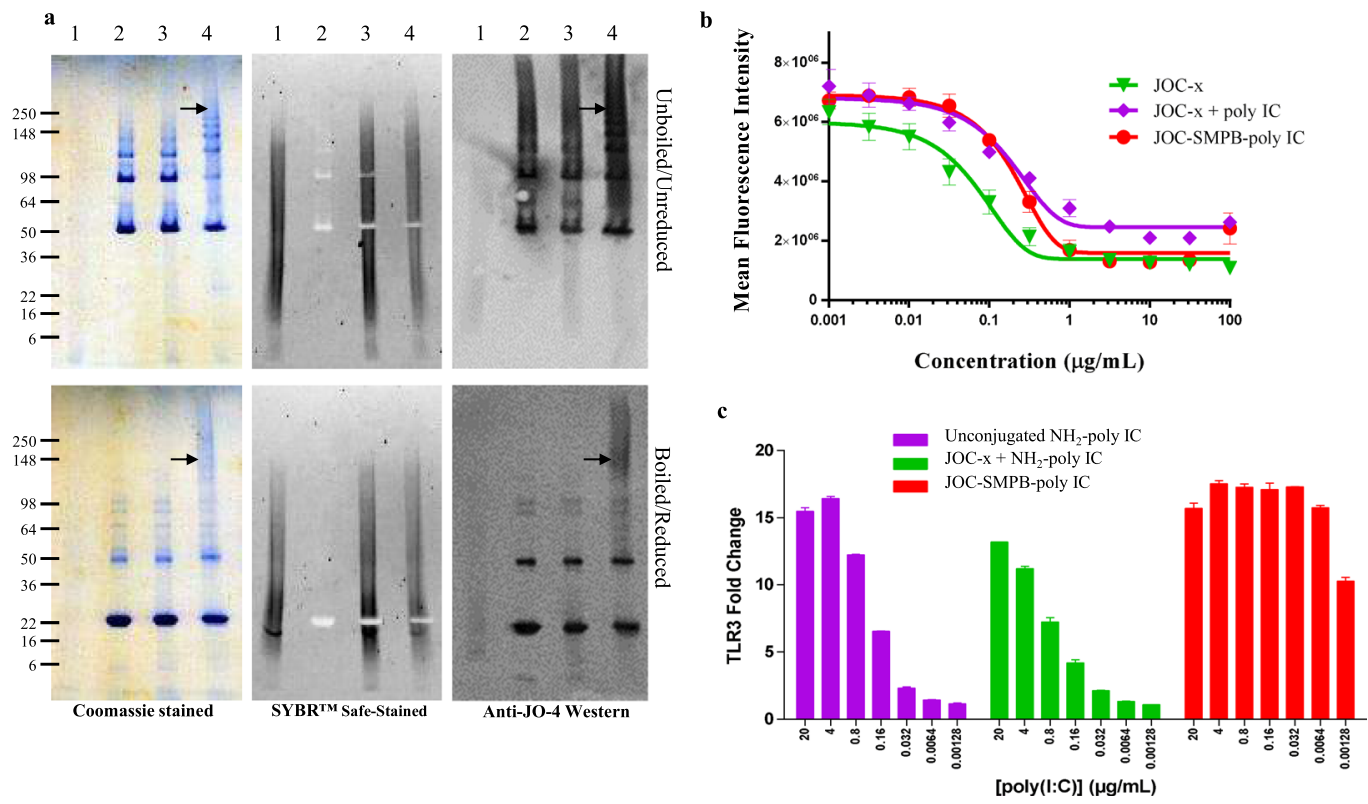

Figure 5. Viral inhibition and TLR3 signaling mediated by JOC-poly(I:C). Panel (a) unboiled/non-reduced and boiled/reduced SDS-PAGE analysis of 1-unconjugated poly(I:C), 2-unconjugated JOC- $x$, 3-a mixture of unconjugated poly(I:C) and JOC- $x$, and 4-JOC-poly(I:C) are shown following imaging by Coomassie staining (left), staining with the DNA intercalating dye SYBR Safe (middle), or following blotting to PVDF and reacting with anti-JO-4 antibodies by Western blot (right). The black arrow denotes the formation of a high MW complex at around $250 \mathrm{kDa}$ in lane 4 (JOC-poly(I:C) conjugates) of all three panels proving the successful conjugation of poly(I:C) to JOC- $x$. Panel (b) viral inhibition curves of unconjugated poly(I:C). The $x$-axis represents the concentration of JOC protein in $\mu \mathrm{g} / \mathrm{mL}$ while the $y$-axis represents the mean fluorescence intensity resulting from entry of reporter virus. A mixture of unconjugated poly(I:C) and JOC- $x$, and the JOC-poly(I:C) conjugate are shown indicating that the JOC-poly(I:C) was able to inhibit DSG2-mediated viral entry, albeit at a slightly lower concentration; Panel (c) shows the fold TLR3 activation mediated by binding of unconjugated $\mathrm{NH}_{4}$-poly(I:C) (purple bars), a mixture of unconjugated $\mathrm{NH}_{4}$-poly(I:C) and JOC- $x$ (green bars), or JOC-poly(I:C) (red bars). Cells were incubated with 7 concentrations of agonist ranging from $20 \mu \mathrm{g} / \mathrm{mL}$ down to $0.00128 \mu \mathrm{g} / \mathrm{mL}$ in five-fold increments.

We demonstrated that the DOTA conjugates maintained their ability to bind DSG2 using the viral inhibition assay (Fig. $4 \mathrm{e}$ ) with $\mathrm{IC}_{50}$ values of $0.055,0.131$, and $0.254 \mu \mathrm{g} / \mathrm{mL}$ for JOC- $x$, JOC-DOTA, and JOC-DOTA (Eu), respectively.

Viral inhibition and TLR3 signaling following poly(I:C) conjugation to JOC- $x$. A final demonstration of the utility of JOC- $x$ was generation of a conjugate that would lead to innate signaling and activation through the viral RNA sensors (TLR3 and MDA5) triggered by the synthetic ligand poly(I:C). Poly(I:C) was conjugated to JOC- $x$ using the heterobifunctional crosslinker SMPB [succinimidyl 4-(p-maleimidophenyl) butyrate] linking the free cysteine on JOC- $x$ to the amino terminus of poly(I:C). When conjugated to JOC- $x$, the poly(I:C) was expected to add an additional 120 to $600 \mathrm{kDa}$ to JOC- $x$ based on the typical size of the poly(I:C) used here which varies between 0.2 and $1.0 \mathrm{~kb}$ in length. Successful conjugation of poly(I:C) to JOC- $x$ is shown in Fig. $5 \mathrm{a}$, in which the black arrows in Lane 4 of the panels identify the JOC-poly(I:C) conjugates which maintain their predictably high molecular weight and conformation even when boiled and reduced (lower 3 panels of Fig. 5a). This conjugated form is not visible in either the unconjugated JOC- $x$ (Lane 2), unconjugated poly(I:C) (Lane 1), or in the unconjugated mix of JOC- $x$ and poly(I:C) (Lane 3) of Fig. 5a. Figure 5b shows the viral inhibition of unconjugated JOC- $x$ (green line, triangles), an equimolar mixture of JOC- $x$ and poly(I:C) (purple line, diamonds), and the JOC-poly(I:C) conjugate (red line, circles). The unconjugated mixture of JOC- $x$ and poly(I:C) was included to measure direct binding of poly(I:C) to HeLa cells given that this cell line has been demonstrated to be TLR3 positive, although TLR3 is primarily found in endosomal compartments and MDA5 in intracellular ${ }^{34}$. In the presence of poly(I:C), unconjugated JOC- $x$ exhibited a $~ 3.9$-fold loss of viral inhibition suggesting possible binding competition. JOC-poly(I:C) conjugates maintained their ability to bind DSG2 and inhibit viral entry, although the slight shift of the inhibition curve to the right is reflective of an approximately 6.3 -fold higher $\mathrm{IC}_{50}$ of the conjugate relative to the unconjugated protein $(0.032 \mathrm{vs} 0.203 \mu \mathrm{g} / \mathrm{mL}$, respectively). To assess whether the JOC-poly(I:C) could bind to TLR3 receptors and induce cell signaling, we utilized an in vitro HEK-Blue human TLR3 reporter cell line (Invivogen, San Diego, CA). As shown in Fig. 5c, we demonstrated that JOC-poly(I:C) conjugates were able to bind to TLR3 and induce signaling at significantly lower protein concentrations compared to poly(I:C) alone or a mixture of JOC- $x$ and poly(I:C). For example, with poly(I:C) alone (purple bars) or a mixture of JOC- $x$ and poly(I:C) (green bars), the two highest concentrations $(20$ and $4 \mu \mathrm{g} / \mathrm{mL}$ ) resulted in $\sim 15$ to 17 -fold upregulation in NF-kB-phosphatase expression, but at the lowest two concentrations $(0.0064$ and $0.00128 \mu \mathrm{g} / \mathrm{mL})$ 


\begin{tabular}{|l|l|l|l|}
\hline Characteristic & JO-4 & GB3 $_{\text {V239D }}$ & GB7 $_{\text {V239D }} /$ JOC- $\boldsymbol{x}$ \\
\hline$p \mathrm{I}$ & 8.91 & 9.11 & 7.30 \\
\hline Production level $(\mathrm{mg} / \mathrm{L})$ & $1-5$ & $1-3$ & $10-15$ \\
\hline Ave. endotoxin $(\mathrm{EU} / \mathrm{mg})$ & $691,300 \mathrm{~K}$ & 80,500 & 2,500 \\
\hline \% aggregated by SEC & $90 / 94 \%$ & $55 / 43 \%$ & $12 / 4 \%$ \\
\hline Affinity to DSG2 (nM) & 0.58 & 0.11 & 1.2 \\
\hline $\begin{array}{l}\text { DSG2 shedding (fold over } \\
\text { PBS treated at 48h) }\end{array}$ & 1.44 & 1.52 & 1.39 \\
\hline VIA IC $_{50}(\mu \mathrm{g} / \mathrm{mL})$ & 0.067 & 0.029 & 0.032 \\
\hline
\end{tabular}

Table 4. Summary of key characteristics of JO4-derivatives. ${ }^{\mathrm{A}}$ First value reported is untreated, second value is following reduction with DTT.

only about a twofold upregulation was seen. Strikingly, at $0.032 \mu \mathrm{g} / \mathrm{mL}$, the JOC-poly(I:C) conjugates (red bars in Fig. 5 c) still exhibited $\sim 18$-fold upregulation compared to either poly(I:C) alone or unconjugated JOC- $x$. In fact, JOC-poly(I:C) maintained $>18$-fold upregulation at every concentration tested down to $0.0064 \mu \mathrm{g} / \mathrm{mL}$, and even at $0.00128 \mu \mathrm{g} / \mathrm{mL}$ still induced a $>10$-fold increase in TLR3 signaling. These results suggest that the JOC-poly(I:C) conjugates may be significantly more potent in stimulating TLR3 signaling than poly(I:C) alone.

\section{Discussion}

Epithelial cancers are characterized by tight junctions that create obstacles to naturally occurring immune cells, antibodies, and drug therapies as well as helping maintain the tumor microenvironment. We have described a new approach to exposing tumors to the action of the immune system and oncological therapy using designer proteins that bind to DSG2 and open tumor tight junctions. Our first generation molecule, JO-1, has been extensively characterized as a co-therapeutic for cancer treatment ${ }^{7,35}$. Binding by JO-1 leads to DSG2 cleavage, shedding, and activation of intracellular signaling pathways which facilitate the opening of junctions. JO-4, a high affinity version of JO-1, has been shown to be well tolerated in vivo and is being developed as a cancer co-therapeutic.

While producing a conjugatable version of JO for targeted cancer therapy we enhanced expression levels through codon optimization and removal of the dimerization domain. We mutated the internal cysteines to serines and found that this did not affect-and may even have enhanced-the activity of the protein as determined by DSG2 binding and viral inhibition. Restoration of a single cysteine was generally sufficient to recover DSG2 binding given that GB5, lacking both cysteines and the DD, failed to form higher MW isoforms and did not bind DSG2, yet still inhibited viral entry - albeit at a much lower level. Both GB7 and GB8, which differ from GB5 by only one cysteine, inhibited viral entry at comparable levels relative to JO-1. As expected, perturbation of the H-I loop involved in DSG2 binding ablated activity. We conclude that the single cysteine may lead to at least trimer-dimer formation resulting in recovery of DSG2 binding. We next down-selected GB3 and GB7 for further analysis of multimerization and aggregation, introduced the affinity-enhancing V239D mutation, and confirmed that the next generation clones $\mathrm{GB}_{\mathrm{V} 239 \mathrm{D}}$ and $\mathrm{GB} 7_{\mathrm{V} 239 \mathrm{D}}$ had retained comparable viral inhibition activity relative to JO-4. SPR analysis confirmed the association and dissociation rates and resulting affinities of GB3 ${ }_{\mathrm{V} 239 \mathrm{D}}$ and $\mathrm{GB7}_{\mathrm{V} 239 \mathrm{D}}$ to hDSG2 were comparable or improved relative to JO-4.

SEC-MALS and TEM analysis of the aggregation state of the proteins revealed that JO-4 exists mainly as higher order structures resembling penton-dodecahedra (Pt-Dd) in both mass and shape. Reduction of JO-4 only minimally disrupted formation of these structures. Removing the cysteines $\left(\mathrm{GB}_{\mathrm{V} 239 \mathrm{D}}\right)$ and dimerization domains $\left(\mathrm{GB}_{\mathrm{V} 239 \mathrm{D}}\right)$ while adding a single terminal cysteine changed the aggregation state of the molecule while providing for an active molecule with the potential for directed attachment of therapeutics. We demonstrated that JO-4, $\mathrm{GB}_{\mathrm{V} 239 \mathrm{D}}$, and GB7 $7_{\mathrm{V} 239 \mathrm{D}}$ exhibited equivalent viral inhibition in the oxidized state, and that only GB7 V239D $_{\text {showed }}$ a marked reduction in viral inhibition upon reduction consistent with the idea that multimerization beyond trimers is required for activity and that the added cysteine provides for this ability.

TEM images of GB7 ${ }_{\mathrm{V} 239 \mathrm{D}}$ revealed very few higher order structures relative to JO-4 as well as the presence of white specks throughout the image. Enhancement of these specks revealed that they were likely trimer-dimers, matching both the shape and predicted size of the trimer-dimers. Presence of these trimer-dimers combined with the comparable viral inhibition of $\mathrm{GB}_{\mathrm{V} 239 \mathrm{D}}$ compared to JO-4 suggest that this protein is still fully able to bind DSG2 and act therapeutically.

Based on physical and biochemical characterization, we down-selected GB7 ${ }_{\mathrm{V} 239 \mathrm{D}}$ for our conjugation experiments and renamed our lead molecule JOC- $x$ to denote that it can open tumor junctions and be conjugated to a payload $(x)$. Primary reasons for choosing GB7 ${ }_{\mathrm{V} 239 \mathrm{D}}$ for our JOC- $x$ included high production yields, low endotoxin binding, and controllable defined aggregation all while retaining similar DSG2 binding and viral inhibition relative to JO-4 (outlined in Table 4).

As proofs-of-concept for this cancer-targeting platform we first sought to generate a reagent useful for pre-targeted immunotherapy (PRIT) and direct tumor imaging by adding pegylated biotins to JOC- $x$. These JOC-PEG-biotin conjugates were active in the viral inhibition assay and were able to bind to SA-Eu for potential use in PRIT applications.

To make a second radiotherapy molecule, we conjugated a metal chelation molecule, DOTA, to JOC- $x$ and demonstrated effective labeling with free europium ions. The JOC-DOTA (Eu) chelates were able to bind DSG2 and inhibit virus in vitro suggesting they will bind to tumor cells in vivo and the metal chelate can be used to irradiate or mark the tumor for in vivo imaging. 
Finally, we generated a potent JOC-poly(I:C) conjugate which also bound DSG2, although with a $\sim 6.3$-fold lower $\mathrm{IC}_{50}$ compared to JOC- $x$ alone in our viral inhibition assay. The reason for the lower $\mathrm{IC}_{50}$ may be due to either impaired kinetics of the conjugate, possibly due to steric hindrances imposed by the high MW of the poly(I:C) or may be a competitive effect caused by poly(I:C) binding to TLR3 present in the HeLa cells themselves. Despite this lower level of binding in the VIA assay, the JOC-poly(I:C) induced 10- to 18-fold upregulation of TLR3 signaling using a HEK Blue TLR3 reporter cell line at every concentration tested down to $0.00128 \mu \mathrm{g} / \mathrm{mL}$. In this assay, poly(I:C) alone only resulted in a 6-fold upregulation of TLR3 signaling at a concentration that was 125 times higher $(0.16 \mu \mathrm{g} / \mathrm{mL})$ than the JOC-poly(I:C). These results suggest that the JOC-poly-IC conjugates are significantly more potent in stimulating double stranded RNA sensor signaling than poly(I:C) alone. Given the dual-targeting of JOC- $x$ to DSG2 and poly(I:C) to TLR3, we predict our JOC-poly(I:C) may exhibit dose sparing qualities by resulting in less non-specific binding of poly(I:C) to non-tumor TLR3 cells as well as targeting the host-directed therapeutic to tumors.

The goal of this study was to develop novel tumor targeting proteins that could be directly coupled to anti-tumor effector molecules. The lead molecule, JOC- $x$, exhibited enhanced biophysical properties with respect to production yields, aggregation, and low residual endotoxins following purification while maintaining high affinity binding to the target receptor DSG2. To demonstrate the versatility of JOC- $x$, we report here DSG2 binding and viral inhibition following conjugation to pegylated biotin, streptavidin-europium, DOTA-europium, and poly(I:C). We also demonstrated that JOC-poly(I:C) induces potent TLR3 activation by inducing $>10$-fold upregulation at concentrations that are $\sim 625$ fold lower than poly(I:C) alone. This effect is likely due to dual targeting and internalization of the conjugate, that is by JOC targeting the conjugate to the cells via DSG2 and activation of double stranded RNA sensors TLR3 and MDA5. We hypothesize that in vivo our JOC-poly(I:C) would likely exhibit enhanced potency by directing poly(I:C) to the tumor while at the same time minimizing non-specific activation of TLR3 receptors on healthy cells. Our JOC- $x$ conjugates are primed for cGMP manufacture and hold much promise for upcoming validation and clinical studies.

\section{Methods}

Cloning and production of JO-1 derivatives. All genes used for this study were designed in silico using the software Serial Cloner v2.4.1. First generation JO derivatives, designated GB1-10, were based on the original JO-1 sequence as described previously ${ }^{7,35}$. The plasmid pET29a (Novagen) was linearized for Gibson cloning using outward facing primers pET29a_GB_UB (5'-TCCTCTCATATGTATATCTCCTTC) and pET29_GB_DT (5'-CTTAATTAGCTGAAATCACTAGT) using a 1/10000 dilution of plasmid as template in the PCR reaction. GB sequences were appended with nucleotides corresponding to the overlap regions of pET29a entry vector and were synthesized as gBlocks ${ }^{\mathrm{TM}}$ by Integrated DNA Technologies (Coralville, IA). Gibson cloning of gBlocks into the pET29a entry vector was performed using the Gibson Cloning Master Mix (New England Biolabs, Ipswich, MA) per manufacturer's instructions. Cloned GBs were sequence confirmed (Genewiz, South Plainfield, NJ) and produced in E. coli Rosetta BL21 (DE3) (EMD Millipore, Darmstadt, Germany).

rhDSG2 binding Western blot. One microgram of junction opener proteins or derivatives were resolved by non-reduced and non-boiled SDS-PAGE using 4-20\% Tris-glycine gradient gels. Proteins were transferred to a polyvinylidene fluoride (PVDF) membrane and blocked with blocking buffer [ $1 \times$ TBS supplemented with $0.05 \%$ Tween-20 (TBST) and 10\% nonfat dry milk (NFDM)] for $2 \mathrm{~h}$ at RT. Membranes were incubated with a $0.75 \mu \mathrm{g} /$ $\mathrm{mL}$ solution of rhDSG2 (Leinco Technologies, Inc., Fenton, MO) in blocking buffer for $1 \mathrm{~h}$ at RT. Following three 10 min washes with TBST, the membrane was incubated with a 1/2000 dilution of murine anti-human DSG2 monoclonal Antibody 6D8 (Bio-Rad, Hercules, CA) in blocking buffer for $1 \mathrm{hr}$ at RT. For detection, the membrane was incubated with a 1/2000 dilution of goat-anti-mouse conjugated to horseradish peroxidase (Southern Biotech, Birmingham, AL) in blocking buffer for $1 \mathrm{~h}$ at RT. After three washes with TBST and three washes with diH2O, bound HRP was detected using TMB Stabilized Substrate for Horseradish Peroxidase (Promega, Madison, WI).

Viral inhibition assay. HeLa cell suspensions were confirmed to be $>98 \%$ viable, their concentration adjusted to $1 \times 10^{5}$ cells $/ \mathrm{ml}$, and then plated in 96 well plates with $200 \mu \mathrm{L}$ per well (Corning, Inc., Corning, NY). Following $18 \mathrm{~h}$ incubation at $37^{\circ} \mathrm{C}, 5 \% \mathrm{CO}_{2}$, growth media was discarded and replaced with $62.5 \mu \mathrm{L}$ of protein in complete Dulbecco's Modified Eagles Medium (cDMEM) [cDMEM=DMEM supplemented with $10 \%$ Fetal Bovine Serum, $2 \mathrm{mM}$ Glutamax, and $100 \mathrm{U} / \mathrm{mL}$ penicillin and $100 \mathrm{ug} / \mathrm{mL}$ Streptomycin (all components from Gibco)]. A total of 11 half-log dilutions were tested in quadruplicate for each protein. Following $1 \mathrm{~h}$ incubation, $50 \mu \mathrm{L}$ of Ad3-GFP virus in complete DMEM was added at a Multiplicity of Infection (MOI) of 100. Two hours later, media was removed and replaced with fresh DMEM, and the plates incubated at $37^{\circ} \mathrm{C}$ and $5 \% \mathrm{CO}_{2}$. The following day, GFP fluorescence was measured from the bottom read orientation at $475 \mathrm{~nm}$ Excitation and $505 \mathrm{~nm}$ Emission using a SpectraMax i3x plate reader (Molecular Devices, Inc., Sunnyvale, CA). Data were plotted using Graphpad Prism 7 (GraphPad Software, Inc., La Jolla, CA) and $\mathrm{IC}_{50}$ values determined using a 5-parameter non-linear fit of the sigmoidal curves in Softmax Pro software (Molecular Devices).

SPR analysis of binding affinities to rhDSG2. SPR analysis was performed at $25^{\circ} \mathrm{C}$ using a Biacore 3000 instrument (GE Healthcare, Pittsburgh, PA). For immobilization of DSG2, rhDSG2 $(10 \mu \mathrm{g} / \mathrm{ml})$ was captured on a CM5 sensor chip using amine coupling chemistry until a coupling level of $6000 \mathrm{RU}$ was obtained. GB7 $\mathrm{V} 239 \mathrm{D}_{\mathrm{D}}$ and $\mathrm{GB}_{\mathrm{V} 239 \mathrm{D}}$ binding were measured in $10 \mathrm{mM}$ HEPES, $150 \mathrm{mM} \mathrm{NaCl}, 0.005 \%$ surfactant P20, $2 \mathrm{mM} \mathrm{CaCl}_{2} \mathrm{pH}_{7.4}$ at a flow rate of $15 \mu \mathrm{l} / \mathrm{min}$. A total of three dilutions of $\mathrm{GB}_{\mathrm{V} 239 \mathrm{D}}$, ranging from $19 \mathrm{nM}$ to $9.5 \mathrm{nM}$, and 4 dilutions of $\mathrm{GB}_{\mathrm{V} 239 \mathrm{D}}$, ranging from $8.3 \mathrm{nM}$ to $0.83 \mathrm{nM}$, were tested. For immobilization of junction opener proteins, JO-4 and $\mathrm{GB}_{\mathrm{V} 239 \mathrm{D}}$ were diluted to $10 \mu \mathrm{g} / \mathrm{ml}$ in $10 \mathrm{mM}$ sodium acetate $\mathrm{pH} 4.5$ and immobilized on a CM5 sensor 
chip (approximately $6000 \mathrm{RU}$ ) by standard amine coupling chemistry. Human rhDSG2 binding was measured in $10 \mathrm{mM}$ HEPES, $150 \mathrm{mM} \mathrm{NaCl}, 0.005 \%$ surfactant P20, $2 \mathrm{mM} \mathrm{CaCl}_{2} \mathrm{pH} 7.4$ at a flow rate of $15 \mu \mathrm{l} / \mathrm{min}$. A total of 5 dilutions of rhDSG2, ranging from $17.4 \mathrm{nM}$ to $0.87 \mathrm{nM}$, were tested in triplicate. The surfaces were regenerated by pulse injection of $5 \mathrm{mM}$ EDTA. The signal recorded on a reference flow cell without protein was subtracted from those signals obtained on protein. For association data, proteins were injected at a flow rate of $15 \mu \mathrm{L} / \mathrm{min}$ for 180 seconds. Following the association phase, buffer was flowed at $15 \mu \mathrm{L} / \mathrm{min}$ for an additional 150 seconds to measure dissociation. Binding curves were analyzed using BIAEvaluation software (GE Healthcare) and data was fit to a 1:1 Langmuir interaction model. Affinities were mathematically derived by dividing the dissociation rate by the association rate.

Size-exclusion chromatography and multi-angle light scattering analysis (SEC-MALS). Size-exclusion chromatography was performed on an Agilent 1200 HPLC system (Agilent Technologies, Santa Clara, CA) with GE HealthCare Superdex 200 Increase 10/300 GL prepacked column and $1 X$ TBS at $1 \mathrm{~mL} / \mathrm{min}$. JO-4, GB3 ${ }_{\mathrm{V} 239 \mathrm{D}}$, and GB7 $7_{\mathrm{V} 239 \mathrm{D}}$ were at concentrations of $2.5 \mathrm{mg} / \mathrm{mL}, 2.8 \mathrm{mg} / \mathrm{mL}$, and $2.5 \mathrm{mg} / \mathrm{mL}$ in PBS with $5 \%$ glycerol, respectively, with $100 \mu \mathrm{L}$ of each injected. Protein was measured via UV absorbance at $280 \mathrm{~nm}$ and sizes determined based on multi-angle light scattering (MALS), with concentration determined using differential refractive index. Analyses were performed using a miniDAWN TREOS MALS detector and Optilab T-rEX differential refractometer using ASTRA software (Wyatt Technology Corporation, Santa Barbara, CA), with the weighted average of the molecular weight reported in $\mathrm{g} / \mathrm{mol}(\mathrm{Mw})$. For reduction of proteins, DTT was added to an initial concentration of $1.6 \mathrm{mM}$, proteins were incubated at $50^{\circ} \mathrm{C}$ for $10 \mathrm{~min}$, and then an additional $1.6 \mathrm{mM}$ DTT was added to maintain the reduced conformations throughout the SEC-MALS analysis.

Transmission electron microscopy. Recombinant GB proteins were visualized by negative-stain electron microscopy. Standard mica-carbon preparations were used with protein at $0.1 \mathrm{mg} / \mathrm{ml}$. Samples were stained using $2 \%$ (wt/vol) uranyl acetate and visualized on a JEOL (JEM-1200EXII) electron microscope at $100 \mathrm{kV}$. Images were acquired and analyzed by digital micrograph software (Gatan).

Conjugation of JOC- $x$ to PEG-biotin or DOTA and labeling with europium. JOC- $x$ was conjugated to both $\mathrm{PEG}_{2}$-biotin and $\mathrm{PEG}_{11}$-biotin using the EZLink ${ }^{\mathrm{TM}}$ Maleimide-PEG2-Biotin and EZLink ${ }^{\mathrm{TM}}$ Maleimide-PEG11-Biotin kits respectively (ThermoFisher Scientific, Waltham, MA, USA). JOC-x protein was first reduced by adding dithiothreitol (DTT) at a concentration of $10 \mathrm{mM}$ and incubated at $37 \mathrm{C}$ for 1 hour. DTT was then removed by running the solution through Zeba desalting columns (ThermoFisher Scientific, Waltham, MA, USA). The maleimide- PEG $_{2 / 11}$-Biotin was then added at a molar ratio of 20:1 (biotin:JOC- $x$ ) and allowed to react, rotating for 24 hours at room temperature. Excess $\mathrm{PEG}_{2 / 11}$-Biotin was then removed, again by Zeba desalting columns. Confirmation of biotin conjugation was subsequently carried out by SDS-PAGE analysis. LanthaScreen ${ }^{\mathrm{TM}}$ Eu-Streptavidin (ThermoFisher Scientific, Waltham, MA, USA) was used as the streptavidin-europium (SA-Eu) payload. JOC-Biotin and the SA-Eu were mixed at a molar ratio of 1:1 in solution, allowed to bind at room temperature for 30 minutes, and then were subsequently run on a SDS-PAGE gel. The gel was then transferred to a PVDF membrane and imaged using the SpectraMax i3x imager (Molecular Devices, San Jose, CA, USA), at an emission wavelength of $616 \mathrm{~nm}$.

For DOTA conjugation to JOC- $x$, JOC- $x$ was reduced by adding DTT at a concentration of $0.1 \mu \mathrm{g} / \mathrm{mL}$ and incubated at $37^{\circ} \mathrm{C}$ for 1 hour. DTT was then removed by running the solution through Zeba desalting columns. Maleimido-mono-amide-DOTA (Macrocyclics, Dallas, TX, USA) was then added to JOC-x at a molar ratio of 10:1 (DOTA:JOC-x), and allowed to react at room temperature overnight. Confirmation of DOTA conjugation to JOC-x was carried out by SDS-PAGE and mass spectrometry. Europium ions were sourced from $\mathrm{EuCl}_{2} \cdot 6 \mathrm{H}_{2} \mathrm{O}$ (Sigma-Aldrich, St. Louis, MO, USA). The JOC-DOTA was buffer exchanged into an ammonium acetate buffer at $\mathrm{pH} 5.8$ to promote chelation of Eu. EuCl $\cdot 6 \mathrm{H}_{2} \mathrm{O}$ was then added at a molar ratio of $10: 1\left(\mathrm{EuCl}_{2} \cdot 6 \mathrm{H}_{2} \mathrm{O}: J O C-D O T A\right)$ and allowed to react at $37^{\circ} \mathrm{C}$ for 1 hour and left at room temperature for 24 hours. $10 \times$ molar excess (of Eu added) of ethylenediaminetetraacetic acid (EDTA) was then added to chelate and remove free Eu. This was followed by running the solution through a Zeba desalting column (ThermoFisher Scientific, Waltham, MA, USA) to eliminate residual europium and to restore the protein buffer to a phosphate $\mathrm{pH} 7.0$ buffer. Confirmation of Eu chelation was done by resolving the proteins on SDS-PAGE, transferring to PVDF membrane, and imaging phosphorescence using a SpectraMax i3X (Molecular Devices, San Jose, CA, USA) at an emission wavelength of $616 \mathrm{~nm}$.

Conjugation of JOC-x to poly $(I: C)$ and measurement of TLR3 signaling. Low molecular weight (LMW) poly(I:C) (Invivogen) was resuspended to a final concentration of $10 \mathrm{mg} / \mathrm{mL}$ in $10 \mathrm{mM}$ sodium phosphate, $150 \mathrm{mM} \mathrm{NaCl}, 10 \mathrm{mM}$ EDTA, $\mathrm{pH}$ 7.2. Next, $0.75 \mathrm{~mL}$ of LMW poly(I:C) was mixed with $125 \mathrm{mg}$ of 1-ethyl-3-(3-dimethylaminopropyl)carbodiimide (EDC) in a 15-mL Falcon tube. Finally, $0.5 \mathrm{~mL}$ of $250 \mathrm{mM}$ ethylenediamine in $100 \mathrm{mM}$ imidazole was added to the poly(I:C), thoroughly vortexed, and $2 \mathrm{~mL}$ of $100 \mathrm{mM}$ imidazole was added and allowed to react overnight at RT. The following day, unreacted EDC and ethylenediamine were removed from the poly(I:C)-NH2 by two passes through $5 \mathrm{~mL} 7 \mathrm{k}$ MWCO Zeba ${ }^{\mathrm{TM}}$ Spin Desalting Columns (Thermo Scientific) that were equilibrated with $100 \mathrm{mM}$ sodium phosphate, $150 \mathrm{mM} \mathrm{NaCl}, 5 \mathrm{mM}$ EDTA, pH 7.2. Purified poly(I:C)-NH2 was then incubated with a 100-fold molar excess of sulfosuccinimidyl 4-(N-maleimidophenyl)butyrate (sulfo-SMPB) (Thermo Scientific) for 1 hour at RT before removing excess sulfo-SMPB with two passes through Zeba ${ }^{\mathrm{TM}}$ columns as described above. Simultaneously, JOC- $x$ was incubated with $5 \mathrm{mM}$ tris(2-carboxyethyl)phosphine (TCEP) for 30 minutes at RT before removing excess TCEP with two passes through Zeba ${ }^{\mathrm{TM}}$ columns. Finally, poly(I:C)-NH2 was incubated with JOC- $x$ at a 1:2 molar ratio at RT overnight. Conjugation was confirmed by nucleic acid gel electrophoresis, SDS-PAGE, and Western blot. 
TLR3 signaling was measured using a commercial HEK-Blue ${ }^{\mathrm{TM}}$ hTLR3 Assay (Invivogen, San Diego, CA) according to manufacturer's specifications. TLR3 activation resulted in NFKB- and AP1-induced production of the reporter gene Secreted Embryonic Alkaline Phosphatase (SEAP).

\section{Data Availability}

Upon publication of the manuscript, we will provide reasonable amounts of requested material following execution of a Material Transfer Agreement for the purposes of replicating data in the paper. The junction opener technology in this manuscript is protected by patents held by University of Washington.

\section{References}

1. Green, S. K., Karlsson, M. C., Ravetch, J. V. \& Kerbel, R. S. Disruption of cell-cell adhesion enhances antibody-dependent cellular cytotoxicity: implications for antibody-based therapeutics of cancer. Cancer Res 62, 6891-6900 (2002).

2. Lavin, S. R., McWhorter, T. J. \& Karasov, W. H. Mechanistic bases for differences in passive absorption. J Exp Biol 210, 2754-2764, https://doi.org/10.1242/jeb.006114 (2007).

3. Lipinski, C. A., Lombardo, F., Dominy, B. W. \& Feeney, P. J. Experimental and computational approaches to estimate solubility and permeability in drug discovery and development settings. Adv Drug Deliv Rev 46, 3-26 (2001).

4. Fessler, S. P., Wotkowicz, M. T., Mahanta, S. K. \& Bamdad, C. MUC1* is a determinant of trastuzumab (Herceptin) resistance in breast cancer cells. Breast Cancer Res Treat 118, 113-124, https://doi.org/10.1007/s10549-009-0412-3 (2009).

5. Oliveras-Ferraros, C. et al. Stem cell property epithelial-to-mesenchymal transition is a core transcriptional network for predicting cetuximab (Erbitux) efficacy in KRAS wild-type tumor cells. J Cell Biochem 112, 10-29, https://doi.org/10.1002/ jcb.22952 (2011).

6. Lee, C. M. \& Tannock, I. F. The distribution of the therapeutic monoclonal antibodies cetuximab and trastuzumab within solid tumors. BMC Cancer 10, 255, https://doi.org/10.1186/1471-2407-10-255 (2010).

7. Beyer, I. et al. Coadministration of epithelial junction opener JO-1 improves the efficacy and safety of chemotherapeutic drugs. Clin Cancer Res 18, 3340-3351, https://doi.org/10.1158/1078-0432.CCR-11-3213 (2012)

8. Christiansen, J. J. \& Rajasekaran, A. K. Reassessing epithelial to mesenchymal transition as a prerequisite for carcinoma invasion and metastasis. Cancer Res 66, 8319-8326, https://doi.org/10.1158/0008-5472.CAN-06-0410 (2006).

9. Lu, Z. Z. et al. Penton-dodecahedral particles trigger opening of intercellular junctions and facilitate viral spread during adenovirus serotype 3 infection of epithelial cells. PLoS pathogens 9, e1003718, https://doi.org/10.1371/journal.ppat.1003718 (2013).

10. Wang, H. et al. Multimerization of adenovirus serotype 3 fiber knob domains is required for efficient binding of virus to desmoglein 2 and subsequent opening of epithelial junctions. J Virol 85, 6390-6402, https://doi.org/10.1128/JVI.00514-11 (2011)

11. Wang, H. et al. Structural and functional studies on the interaction of adenovirus fiber knobs and desmoglein 2 . $J$ Virol 87 , 11346-11362, https://doi.org/10.1128/JVI.01825-13 (2013).

12. Richter, M. et al. Preclinical safety and efficacy studies with an affinity-enhanced epithelial junction opener and PEGylated liposomal doxorubicin. Mol Ther Methods Clin Dev 2, 15005, https://doi.org/10.1038/mtm.2015.5 (2015).

13. Barrios, K. \& Celis, E. TriVax-HPV: an improved peptide-based therapeutic vaccination strategy against human papillomavirusinduced cancers. Cancer Immunol Immunother 61, 1307-1317, https://doi.org/10.1007/s00262-012-1259-8 (2012).

14. Cho, H. I., Barrios, K., Lee, Y. R., Linowski, A. K. \& Celis, E. BiVax: a peptide/poly-IC subunit vaccine that mimics an acute infection elicits vast and effective anti-tumor CD8 T-cell responses. Cancer Immunol Immunother 62, 787-799, https://doi.org/10.1007/ s00262-012-1382-6(2013).

15. Bianchi, F., Pretto, S., Tagliabue, E., Balsari, A. \& Sfondrini, L. Exploiting poly(I:C) to induce cancer cell apoptosis. Cancer Biol Ther 18, 747-756, https://doi.org/10.1080/15384047.2017.1373220 (2017).

16. Muller, E. et al. Toll-Like Receptor Ligands and Interferon-gamma Synergize for Induction of Antitumor M1 Macrophages. Frontiers in immunology 8, 1383, https://doi.org/10.3389/fimmu.2017.01383 (2017).

17. Shime, H. et al. Myeloid-derived suppressor cells confer tumor-suppressive functions on natural killer cells via polyinosinic:polycytidylic acid treatment in mouse tumor models. J Innate Immun 6, 293-305, https://doi.org/10.1159/000355126 (2014).

18. Shime, H. et al. Toll-like receptor 3 signaling converts tumor-supporting myeloid cells to tumoricidal effectors. Proceedings of the National Academy of Sciences of the United States of America 109, 2066-2071, https://doi.org/10.1073/pnas.1113099109 (2012).

19. Durmort, C. et al. Structure of the fiber head of Ad3, a non-CAR-binding serotype of adenovirus. Virology 285, 302-312, https://doi. org/10.1006/viro.2001.0967 (2001).

20. Wang, H. et al. Intracellular Signaling and Desmoglein 2 Shedding Triggered by Human Adenoviruses Ad3, Ad14, and Ad14P1. J Virol 89, 10841-10859, https://doi.org/10.1128/JVI.01425-15 (2015).

21. Lausch, V., Hermann, P., Laue, M. \& Bannert, N. Silicon nitride grids are compatible with correlative negative staining electron microscopy and tip-enhanced Raman spectroscopy for use in the detection of micro-organisms. J Appl Microbiol 116, 1521-1530, https://doi.org/10.1111/jam.12492 (2014).

22. Fender, P., Hall, K., Schoehn, G. \& Blair, G. E. Impact of human adenovirus type 3 dodecahedron on host cells and its potential role in viral infection. J Virol 86, 5380-5385, https://doi.org/10.1128/JVI.07127-11 (2012).

23. Humphrey, W., Dalke, A. \& Schulten, K. VMD: visual molecular dynamics. J Mol Graph 14(33-38), 27-38 (1996).

24. Breitz, H. B. et al. Clinical optimization of pretargeted radioimmunotherapy with antibody-streptavidin conjugate and 90Y-DOTAbiotin. J Nucl Med 41, 131-140 (2000).

25. Domingo, R. J. \& Reilly, R. M. Pre-targeted radioimmunotherapy of human colon cancer xenografts in athymic mice using streptavidin-CC49 monoclonal antibody and 90Y-DOTA-biotin. Nucl Med Commun 21, 89-96 (2000).

26. Green, D. J. et al. Comparative Analysis of Bispecific Antibody and Streptavidin-Targeted Radioimmunotherapy for B-cell Cancers. Cancer Res, https://doi.org/10.1158/0008-5472.CAN-16-0571 (2016).

27. Heppeler, A., Froidevaux, S., Eberle, A. N. \& Maecke, H. R. Receptor targeting for tumor localisation and therapy with radiopeptides. Curr Med Chem 7, 971-994 (2000).

28. Janssen, M. et al. Improved tumor targeting of radiolabeled RGD peptides using rapid dose fractionation. Cancer Biother Radiopharm 19, 399-404, https://doi.org/10.1089/cbr.2004.19.399 (2004).

29. Zhang, W. J. et al. Design, recombinant expression and convenient A-chain N-terminal europium-labelling of a fully active human relaxin-3 analogue. FEBS J 279, 1505-1512, https://doi.org/10.1111/j.1742-4658.2012.08550.x (2012).

30. DeNardo, S. J. et al. Yttrium-90-DOTA-peptide-chimeric L6 radioimmunoconjugate: efficacy and toxicity in mice bearing p53 mutant human breast cancer xenografts. J Nucl Med 39, 842-849 (1998).

31. Deshpande, S. V. et al. Yttrium-90-labeled monoclonal antibody for therapy: labeling by a new macrocyclic bifunctional chelating agent. J Nucl Med 31, 473-479 (1990).

32. Giblin, M. F. et al. In vitro and in vivo comparison of human Escherichia coli heat-stable peptide analogues incorporating the 111InDOTA group and distinct linker moieties. Bioconjug Chem 15, 872-880, https://doi.org/10.1021/bc049974x (2004). 
33. Sosabowski, J. K. \& Mather, S. J. Conjugation of DOTA-like chelating agents to peptides and radiolabeling with trivalent metallic isotopes. Nat Protoc 1, 972-976, https://doi.org/10.1038/nprot.2006.175 (2006).

34. Hasimu, A., Ge, L., Li, Q. Z., Zhang, R. P. \& Guo, X. Expressions of Toll-like receptors 3, 4, 7, and 9 in cervical lesions and their correlation with HPV16 infection in Uighur women. Chin J Cancer 30, 344-350 (2011).

35. Beyer, I. et al. Epithelial junction opener JO-1 improves monoclonal antibody therapy of cancer. Cancer Res 71, 7080-7090, https:// doi.org/10.1158/0008-5472.CAN-11-2009 (2011).

\section{Acknowledgements}

This research was funded by grants from the National Institutes of Health Small Business Grants 1R43CA18337901 and 2R44CA162582-02 awarded to D.C. and A.L. We are grateful to the CNRS for the PICS travelling grant between Grenoble and Seattle. We are grateful to Dr. Jeff Guderian at the Infectious Disease Research Institute for performing the HEK Blue TLR3 signaling assays. We are also grateful to the University of Washington Mass Spectrometry Core Facility for analyzing our JOC-DOTA conjugates.

\section{Author Contributions}

S.A.G., D.C., A.L. and R.P. conceived the experiments. S.A.G., R.P., J.K. and D.C. wrote the manuscript. J.D.-B. performed protein purification and viral inhibition assays. R.P. and L.C. performed SEC and SEC-MALS analysis. Additional MALS analysis was performed by J.A.A. H.W. and J.A. performed DSG2 binding experiments. E.V.-S. and P.F. performed Biacore analysis and TEM imaging. J.K. performed experiments with DOTA and europium labeling. R.P. performed poly(I:C) experiments. S.A.G. and D.C. supervised the project and coordinated research. All authors reviewed the manuscript.

\section{Additional Information}

Supplementary information accompanies this paper at https://doi.org/10.1038/s41598-019-42229-3.

Competing Interests: R.P., J.K., J.D-B., C.T., E.V-S., H.W., J.A., L.C., J.A.A., P.F. and S.A.G. declare no competing interests. D.C. and A.L. are co-owners of Compliment Corp., a start-up company that is involved with the clinical development of the JO technology.

Publisher's note: Springer Nature remains neutral with regard to jurisdictional claims in published maps and institutional affiliations.

(c) () Open Access This article is licensed under a Creative Commons Attribution 4.0 International License, which permits use, sharing, adaptation, distribution and reproduction in any medium or format, as long as you give appropriate credit to the original author(s) and the source, provide a link to the Creative Commons license, and indicate if changes were made. The images or other third party material in this article are included in the article's Creative Commons license, unless indicated otherwise in a credit line to the material. If material is not included in the article's Creative Commons license and your intended use is not permitted by statutory regulation or exceeds the permitted use, you will need to obtain permission directly from the copyright holder. To view a copy of this license, visit http://creativecommons.org/licenses/by/4.0/.

(c) The Author(s) 2019 